\title{
The Study on Antioxidation and Mechanism of 2 - Bromo - 4'- Nitroacetophenone
}

\author{
Runze Han (Corresponding author) \& Dongmei Tian \\ School of Pharmaceutical Engineering and Life Sciences, \\ Changzhou University, Changzhou, China. \\ E-mail: 1090317838@qq.com
}

Received: November 14, 2017 Accepted: December 1, 2017

doi:10.5296/jbls.v9i2.12819 URL: https://doi.org/10.5296/jbls.v9i2.12819

\begin{abstract}
The 2-Bromo-4'-nitroacetophenone is an important compound which is widely used in the modification of methionine residues in alpha-chymotrypsin, organic synthesis materials and pharmaceutical intermediates. Our laboratory recently found that the 2-bromo-4'-nitroacetophenone can extend the lifespan of the C. elegans. Therefore, we want to know whether this compound has the anti-oxidative ability.

In this paper, we used three different concentrations of 2-bromo-4'-nitroacetophenone to feed normal C. elegans and the mutants (daf-16). we found that 2-bromo-4'-nitroacetophenone has antioxidant effect and this effect is dependent on Insulin pathway.
\end{abstract}

Keywords: C. Elegans, 2-bromo-4'-nitroacetophenone, anti-oxidation

\section{Introduction}

Upon stepping into the twenty-first century, how to solve the problem of aging is attracting more and more people's attention. The current majority of the country's population reached or exceeded the total population for more than 10 percent. With the lifting living standard, how to solve the problem of aging will be the arduous task facing mankind(Zhuang et al., 2016; Weiming Zhang et al., 2013). From the biological point of view, aging is the decline of body structure and ability with the time passing by. From the pathological point of view, aging is due to the body's cell state emergency caused damage, resulting in the body's immune response to decline and metabolism (Jiong Wu et al., 2017; Guo SH et al., 2007;).

The essence of aging is the process of making the various tissues and organs of the organism unable to meet the needs of the organism as the age increases. Although aging is the inevitable result of nature ( Nie GJet al., 2002, Gami MS \&Wolkow CA, 2006), it is found 
that the process of aging can be effectively carried out by some biological means (Wilkinson DS et al., 2012; Sangha JS et al., 2012) . In this experiment, we used the C. elegans as a model organism to feed normal $\mathrm{C}$. elegans and mutants with 2-bromo-4'-nitroacetophenone and found that 2-bromo-4'- Nitroacetophenone has antioxidant properties. The mechanism is mainly through the control of the insulin pathway to achieve.

\section{Materials and Methods}

\subsection{Reagents and C. elegans Strain Preparation}

The 2-bromo-4'-nitroacetophenone is obtained from Sigma-Aldrich (St. Louis, MO, USA), and its purities is $95 \%$. It was dissolved in M9 solution and then diluted by M9.

Nematodes used were wild-type C. elegans N2, mutants of daf-16 originally obtained from Caenorhabditis Genetics Center. They were maintained on nematode growth medium (NGM) plates seeded with Escherichia coli OP50 at $20^{\circ} \mathrm{C}$ as described.

Gravid nematodes were washed off the plates into centrifuge tubes, and were lysed with a bleaching mixture $(0.45 \mathrm{M} \mathrm{NaOH}, 2 \% \mathrm{HOCl})$. Age synchronous populations of L1-larvae nematodes were obtained by the collection. Nematodes were washed with a modified $\mathrm{S}$ medium (Ren HR et al,2011; Brenner S, 1974). Exposures were performed from L1-larvae to adult (prolonged exposure) in $\mathrm{K}$ medium of 96-well sterile tissue culture plates at $20^{\circ} \mathrm{C}$ incubator in the presence of food. Paraquat was added forty eighteen hours later ( Donkin $\mathrm{S}$ \& Williams PL, 2012).

\subsection{Locomotion behavior}

To determine the Locomotion behavior of lifespan. Head thrash and body bend were used as endpoints ( Akesson Chet al., 2003). The number of head wobbles and body bending times of Caenorhabditis elegans in the specified time were observed.

During the locomotion behavior assay, the examined nematodes were transferred into the assay plate containing $\mathrm{K}$ medium on top of the agar. After a recovery time of $1 \mathrm{~min}$, head thrashes, and body bends were counted for $1 \mathrm{~min}$ and 20s respectively. (Kim J et al., 2008; Louppis A P et al., 2010).

\subsection{Stress assy}

To assay thermal stress resistance, worms pre-treated with the 2-bromo-4'-nitroacetophenone for 48 -hr were transferred to the $35^{\circ} \mathrm{C}$ condition, and then the lifespan was measured and analyzed as described above. To determine antioxidant activity, paraquat was added to Caenorhabditis elegans pre-treated with 2-bromo-4'-nitroacetophenone for 48 hours, and then the longevity was measured and analyzed as described above ( Honda Y et al., 1999; Sutphin GL et al., 2012) .

\subsection{Statistical analysis}

All data in this article are expressed as mean \pm standard error (S.E.M.). Generate charts using Microsoft Excel (Microsoft Corporation, Redmond, WA). Statistical analysis was performed 
using SPSS 12.0 (SPSS Inc., Chicago, USA). Differences between groups were determined by analysis of variance (ANOVA). Probability levels 0.05 and 0.01 are considered statistically significant.

\section{Results}

\subsection{2-Bromo-4'-nitroacetophenone has antioxidant effect on C. Elegans 。}

The treatment is carried out with 2-bromo-4'-nitroacetophenone throughout the life of the larval stage. We can see the 2-Bromo-4'-nitroacetophenone has an antioxidant effect on C. Elegans(Figure 1).
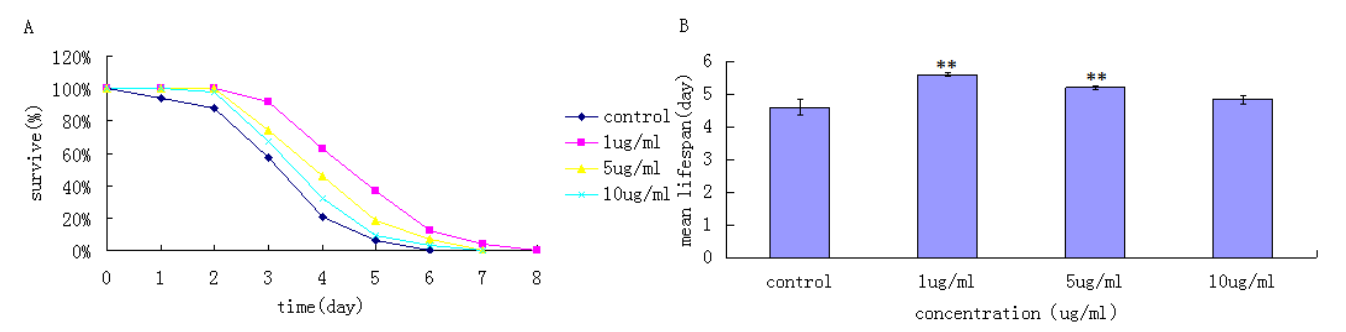

Figure 1. The 2-Bromo-4'-nitroacetophenone has antioxidant properties. (A) Survival curve of nematodes after pretreatment at different concentrations of 2-Bromo-4'-nitroacetophenone in paraquat-containing medium. (B) The average lifespan after treatment of the Caenorhabditis elegans with 2-Bromo-4'-nitroacetophenone in paraquat-containing medium.

$$
(* \mathrm{P}<0.05, * * \mathrm{P}<0.01)
$$

\subsection{The 2-Bromo-4'-nitroacetophenone has improves the locomotion behavior of} Caenorhabditis elegans.

We studied the effects of 2-bromo-4'-nitroacetophenone on the locomotion behavior of C. elegans and found that the nematode treated with 2-bromo-4'-nitroacetophenone significantly improved the motor behavior of C. elegans(Figure 2).
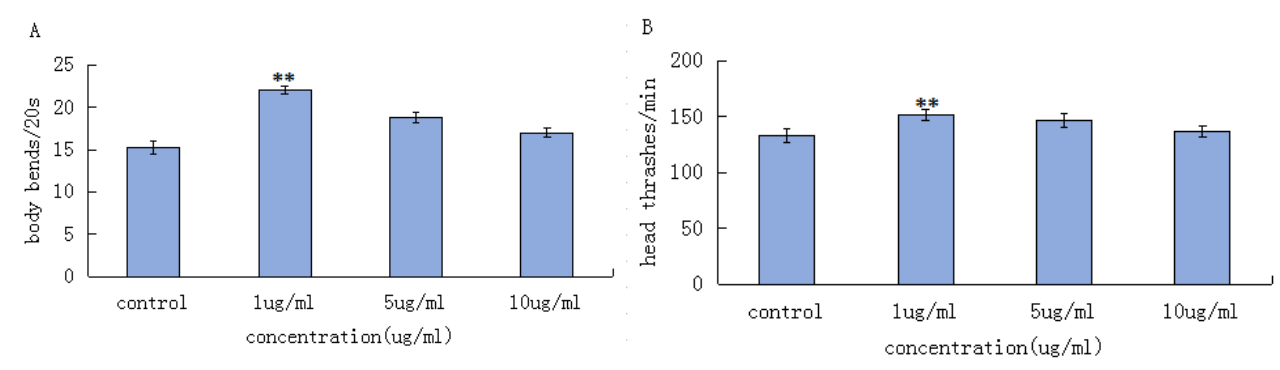

Figure 2. The 2-bromo-4'-nitroacetophenone improves the locomotion behavior and reproductive capacity of Caenorhabditis elegans. (A) Progeny of animals exposed to 2-bromo-4'-nitroacetophenone on locomotion behavior as indicated by head thrashes. (B) Progeny of animals exposed to 2-bromo-4'-nitroacetophenone on locomotion behavior as indicated by body bends. ( $* \mathrm{P}<0.05, * * \mathrm{P}<0.01$ ) 


\section{Macrothink}

3.3 2-bromo-4'-nitroacetophenone improves the heat stress of Caenorhabditis elegans

The C. elegans treated with 2-bromo-4-nitroacetophenone were transferred to a temperature of $35^{\circ} \mathrm{C}$ to determine 2-bromo- $4^{\prime}-$ nitroacetophenone have resistance of C. Elegans. The mean lifespan the effect of C.elegans treated with 2-bromo-4'-nitroacetophenone Increased. Indicating that the 2-bromo-4-nitroacetophenone improved the heat stress of the C. Elegans (Figure 3).
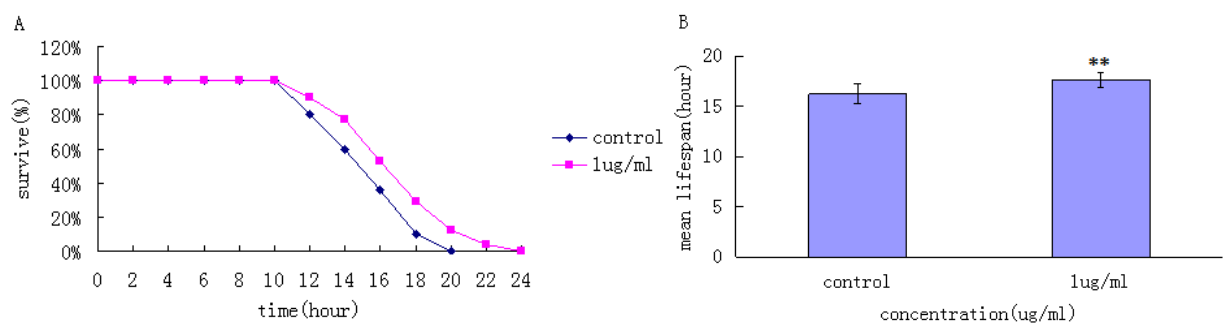

Figure 3. The 2-bromo-4-nitroacetophenone improves the heat stress of caenorhabditis elegans.. (A) Survival curves of C. elegans pretreated with $1 \mathrm{ug} / \mathrm{ml}$ or without of 2-bromo-4'-nitroacetophenone in $35^{\circ} \mathrm{C}$. (B) Mean lifespans of C. elegans pretreated with or without 2-bromo-4'-nitroacetophenone in $35^{\circ} \mathrm{C} .(* \mathrm{P}<0.05, * * \mathrm{P}<0.01)$

3.4 2-bromo-4'-nitroacetophenone has antioxidant effect and this effect is dependent on Insulin pathway

Compared with the control group, the mutants of C. elegans had significantly lower antioxidant activity, indicating that 2-bromo-4'-nitroacetophenone on Caenorhabditis elegans oxidation resistance through control of genes daf-16 to achieve.(Figure 4)(Xu X et al., 2007; I.N. et al 2003).
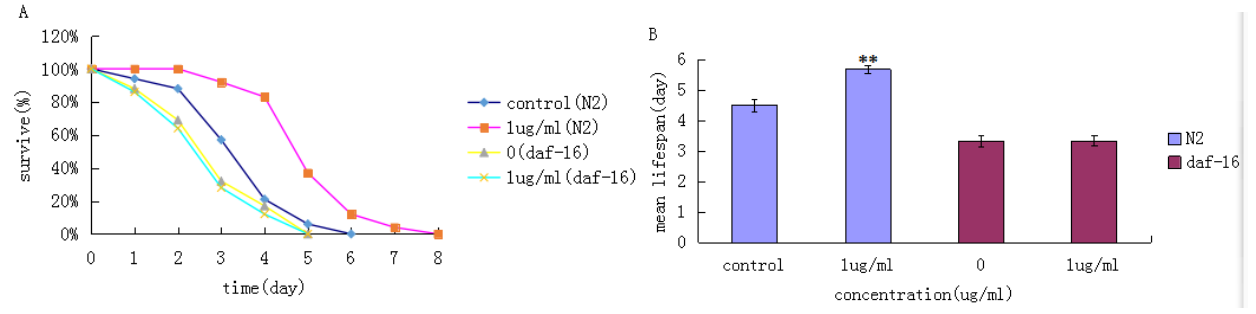

Figure 4.Antioxidant properties in wild-type and mutants exposed to

2-Bromo-4'-nitroacetophenone . (A) Survival curves of N2 and daf-16 pretreated with different densities of 2-Bromo-4'-nitroacetophenone in paraquat-containing medium. (B)

Mean lifespans of N2 and daf-16 pretreated with different densities of 2-Bromo-4'-nitroacetophenone in paraquat-containing medium. $(* \mathrm{P}<0.05$, **P $<0.01)$

Compared with the normal wild-type C. elegans, the transformed body of C. elegans had a much weaker body curvature and head swing. This further confirms our conclusion. (Figure 
$5)$.

In summary, we can demonstrate that daf-16 participates in the antioxidant regulation of $\mathrm{C}$. elegans by mutant daf-16, that is, the antioxidant activity of $\mathrm{C}$. elegans is mainly controlled by genes. (Figure 4 and Figure 5 ).

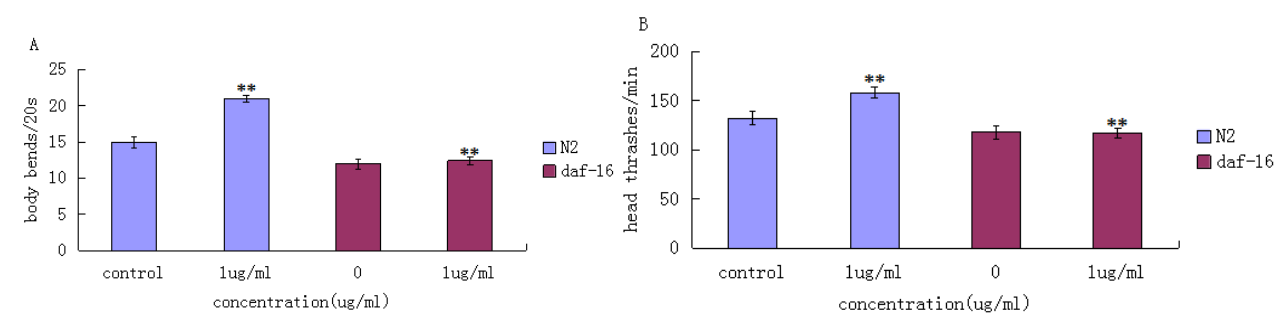

Figure 5. Locomotion behaviors in wild-type and mutants exposed to EP. (A) Effects of 2-Bromo-4'-nitroacetophenone on locomotion behavior in daf-16 and N2 as indicated by body bends. (B) Effects of 2-Bromo-4'-nitroacetophenone on locomotion behavior in daf-16 and $\mathrm{N} 2$ as indicated by head thrashes. $(* \mathrm{P}<0.05, * * \mathrm{P}<0.01)$

\section{Discussion}

In this experiment, we found that 2-bromo-4'-nitroacetophenone had a significant antioxidant effect, and reached its best at $1 \mathrm{ug} / \mathrm{ml}$ concentration [Figure 1]. In addition, compared with the control group, the nematode treated with 2-bromo-4'-nitroacetophenone significantly improved the motility of the Caenorhabditis elegans during the aging process [Figure 2]. These results indicate that 2-bromo-4 '- nitroacetophenone has a potential anti-oxidative effect.

Under the thermal stress conditions, the C.elegans treated with 2-bromo-4'-nitroacetophenone were able to survive for longer periods under the same conditions compared to the control group[Figure 3), the condition of 2-bromo-4 '- nitroacetophenone has the effect of improving heat stess.

As can be seen from Figure 4, under the same conditions, the survival rate of daf-16 nematode is reduced. From the above data, the compound 2-bromo-4'-nitroacetophenone Control of nematode life is achieved mainly through the control of the corresponding genes, in which daf -16 nematode life is of the greatest impact. That is to say 2-bromo-4'-nitroacetophenone has antioxidant effect and this effect is dependent on Insulin pathway.

Correspondingly, after the mutation of the C. elegans, its movement behavior will be correspondingly reduced, and these data further confirmed our conclusion. However, the mechanism of controlling the life of nematodes is very complex and requires more experiments to verify. 


\section{References}

Akesson Ch, Pero RW, Ivars F. C-Med 100, a hot water extract of Uncaria tomentosa, prolongs lymphocyte survival in vivo. [M]. Phytomedicine: international journal of phytotherapy and phytopharmacology 2003, 1(1).

Bonafe M, Barbieri M, Marchegiani F, Olivieri F, Ragno E, Giampieri C, Mugianesi E, Centurelli M, Franceschi C, Paolisso G. Polymorphic variants of insulin-like growth factor I (igf-I) receptor and phosphoinositide 3-kinase genes affect igf-I plasma levels and human longevity: cues for an evolutionarily conserved mechanism of life span control. [J]. The Journal of Clinical Endocrinology and Metabolism 2003, 7(7).

Brenner S (1974) The genetics of Caenorhabditis elegans. Genetics 77:71-94.

C. Li, H. Sun, A. Chen, X. Ning, H. Wu, S. Qin, et al (2014) .Identification and characterization of an intracellular $\mathrm{Cu}, \mathrm{Zn}$-superoxide dismutase (icCu/Zn-SOD) gene from clam Venerupis philippinarum.

Donkin S, Williams PL(2012). Influence of developmental stage, salts and food presence on various end points usingCaenorhabditis elegans for aquatic toxicity testing. Environ Toxicol Chem.

Gami MS, Wolkow CA. Studies of Caenorhabditis elegans DAF-2/insulin signaling reveal targets for pharmacological manipulation of lifespan. [J]. Aging cell 2006, 1(1).

Guo SH, Yah JQ, Bezard E, Yang TB, Yang XQ, Zhao BL. Protective effects of green tea polyphanols in the 6-OHDA rat model of Parkinson's disease through inhibition of ROS-NO pathway[J]. Biojogical Psychiatry 2007, 62.

Honda, Y, Honda, S. The daf-2 gene network for longevity regulates oxidative stress resistance and Mn-superoxide dismutase gene expression in Caenorhabditis elegans. [M]. The FASEB Journal 1999, 11(11).

Hsu AL, Murphy CT, Kenyon C. Regulation of aging and age-related disease by DAF-16 and heat-shock factor. Science. 2003 May 16;300:1142-5.

I.N. Zelko, T.J. Mariani, R.J. FolzSuperoxide dismutase multigene family: a comparison of the CuZn-SOD (SOD1), Mn-SOD (SOD2), and EC-SOD (SOD3) gene structures, evolution, and expression Free Radic Biol Med, 33 (2002), pp. 337-349

Jiong Wu, Miaomiao Bao, Delong Ge, Liping Huo, Zhenming Lv, Changfeng Chi, Zhi Liao, Huihui Liu. "The expression of superoxide dismutase in Mytilus coruscus under various stressors", Fish \& Shellfish Immunology, 2017

Kim J, Takahashi M, Shimizu T, Shirasawa T, Kajita M, Kanayama A, Miyamoto Y. [J]. Mechanisms of Ageing and Development 2008.

Lithgow G, White T, Melov S, Johnson T (1995) Thermotolerance and extended life-span conferred by single-gene mutations and induced by thermal stress. Proc Natl Acad Sci USA 92:7540-7544. 


\section{Al Macrothink}

Journal of Biology and Life Science ISSN 2157-6076 2018, Vol. 9, No. 2

Louppis A P, Badeka A V, Katikou P, et al. Determination of okadaic acid, dinophysistoxin-1 and related esters in Greek mussels using HPLC with fluorometric detection, LC-MS/MS and mouse bioassay. Toxicon, 2010, 55 (4): 724-733.

Nie GJ. , Jin CF., Cao YL., Shen SR., Zhao BL. . Distinct effects of tea catechins on 6-hydroxydopamine-induced apoptosis in PC12 cells[J]. Archives of Biochemistry and Biophysics 2002, 1(1).

Qian $\mathrm{H}, \mathrm{Xu} \mathrm{X}$. Reduction in DNA methyltransferases and alteration of DNA methylation pattern associate with mouse skin ageing. Exp Dermatol. 2014 May;23:357-9.

Ren HR, Shan CY, Jiang HF, Zhang WM (2011) Inhibition of the extract from Nymphaea hybrid on the activity of tyrosinase (in Chinese). Nat Prod Res and Dev 23(6):1122-1126 (ISSN: 1001-6880).

Sangha JS, Sun X, Wally OS, Zhang K, Ji X, Wang Z, et al. Liuwei Dihuang (LWDH), a traditional Chinese medicinal formula, protects against $\beta$-amyloid toxicity in transgenic Caenorhabditis elegans. PLoS One.2012;7:e43990.

Weiming Zhang, Ting Lv, Min Li, Qiuli Wu, Linsong Yang, Hui Liu, Dafeng Sun, Lingmei Sun, Ziheng Zhuang, Dayong Wang. "Beneficial Effects of Wheat Gluten Hydrolysate to Extend Lifespan and Induce Stress Resistance in Nematode Caenorhabditis elegans", PLoS ONE, 2013

Wilkinson DS, Taylor RC, Dillin A. Analysis of aging in Caenorhabditis elegans. Methods Cell Biol.2012;107:353-81.

Xu X, Zhan M, Duan W, Prabhu V, Brenneman R, Wood W, Firman J, Li H, Zhang P, Ibe C, Zonderman AB, Longo DL, Poosala S, Becker KG, Mattson MP. Gene expression atlas of the mouse central nervous system: impact and interactions of age, energy intake and gender. Genome Biol. 2007;8:R234.

Zhang W, Lv T, Li M, Wu Q, Yang L, Liu H, et al. Beneficial effects of wheat gluten hydrolysate to extend lifespan and induce stress resistance in nematode Caenorhabditis elegans. PLoS One. 2013;8:e74553.

Zhuang, Ziheng, Ting Lv, Min Li, Yusi Zhang, Ting Xue, Linsong Yang, Hui Liu, and Weiming Zhang. (2014). Zhuang, Ziheng, Ting Lv, Min Li, Yusi Zhang, Ting Xue, Linsong Yang, Hui Liu, and Weiming Zhang. "The Lifespan-Extending Effects of Nymphaea hybrid Root Extract in the Nematode Caenorhabditis elegans", Plant Foods for Human Nutrition, 2014.

1. Donkin, S. G., \& Dusenbery, D. B. (1993). A soil toxicity test using the nematode Caenorhabditis

2. elegans and an effective method of recovery. Arch Environ Contam To 


\section{Copyright Disclaimer}

Copyright for this article is retained by the author(s), with first publication rights granted to the journal.

This is an open-access article distributed under the terms and conditions of the Creative Commons Attribution license (http://creativecommons.org/licenses/by/3.0/). 